\title{
Benefits and Disadvantages of Assay Kits in Experimental Education
}

\author{
Yu Yun ${ }^{1, a}$ Yi Zhang ${ }^{2, b}$ and Weigang Duan ${ }^{2, c}$ \\ ${ }^{1}$ School of Basic Medicine, Kunming Medical University, 1168 West Chunrong Road, Kunming \\ 650500, China \\ ${ }^{2}$ School of Pharmaceutical Science, Yunnan University of Traditional Chinese Medicine, 1076 Yuhua \\ Road, Kunming 650500, China \\ ayunyu_li@126.com, ${ }^{b} 574378413 @ q q . c o m,{ }^{c}$ deardwg@126.com
}

\begin{abstract}
Keywords: assay kits; experimental education; college education; benefits; disadvantages
Abstract. Experimental education is an essential part of natural science education system in college. Recently, application of various assay kits becomes popular in experimental education. The kits make the experimental operation simpler and more efficient with much better experiment results. However, the utility of the kits increases experimental costs, and could hamper teaching skill improvement for experiment teachers and hamper practical ability enhancement for students. Although some of these disadvantages are manageable, it is strongly suggested that the kits are only an alternative, not suitable for all experimental education programs.
\end{abstract}

\section{Introduction}

Experimental education is an important element in natural science education system in college, facilitating quality-oriented education and personnel training, and is beneficial for student to develop good scientific quality, innovation thoughts, good practice ability, and good abilities in problem analyzing and solving [1,2]. Also, experimental education is the key component of curriculum teaching in natural sciences. Experimental education is able to consolidate the knowledge learnt from theory education, enhance their practice skills, and arouse their enthusiasm of learning [3]. The experimental education can be carried out anywhere in any forms [4, 5]. Recently, kits are becoming more and more common in college laboratories for experimental education. However, some disadvantages emerged that were not recognized before.

\section{The feature of assay kits}

An assay kit is constituted by several reagents and materials to conduct an expected assay task conveniently with expected results under its standard operation protocol for experimenters. The reagents in the kit is prepared ready for direct use, or only need one or more steps of dissolution or dilution before use. The materials are also prepared for direct use, or only need one or several steps of connection or installation. The standard operation protocol gives the optical steps and conditions in detail for the experimenters. The protocol is expressed so simple that a common student can perform it even without special training. According to the aim of design, there are kits for education, for scientific research and for clinic. Although some assay kits for education have been designed and are purchasable in markets [6, 7], they are not popular in laboratories. In fact, the kits for scientific research are flooding the reagent markets. Here, we will mainly discuss the disadvantages of kits (for scientific research) in experimental education, besides their benefits.

In consideration of simplification or some commercial aspects, the reagents in the kits usually tagged as "Solution 1", "Solution 2", "Buffer 1", "Buffer 2", etc. without further explanation. The kits are even unwillingly to tell the chemicals the reagents contained and the formulae of the solution. The materials usually marked as "affinity column", "centrifuge tube", etc. The kit is unwillingly to inform the physical and chemical properties of the materials either [8]. Even further, many assay kits may not explain their principles. The information most addressed is its function. In order to insure the experimenter to harvest the expected results, the steps and the conditions are presented in detail as possible as it can. 
Therefore, what an assay kit pursues are convenience, accuracy, and efficiency. The object they aim at is scientific research, not experimental education. The kits may pay more attention on the convenient operability than educational purpose, and inevitably will cause some disadvantages when used for experimental education.

\section{The disadvantages of assay kits in experimental education}

The most obvious benefits are time saving and preparation simplifying as for a teacher to prepare the experiment. The students will also feel some benefits, such as procedure simplification and result insurance. What the students should do is to follow the steps offered by the standard operation protocol. Therefore, an experiment that could not be finished in three hours before can be finished in less than two hours. With efficiency enhancing, the assay kits are welcome by teachers and students.

However, there are disadvantages of kits in the whole progress of experimental education. They are listed below.

Increasing experiment cost. A kit is usually more expensive than self-made reagents concocted from the same formulae. The kit even cost ten times more than the self-made reagents. For example, the self-made reagents for plamid extraction based on alkaline lysis principle $[9,10]$ cost about 100 $\mathrm{RMB}$, which are enough for 30 students to complete their experiment. If domestic commercial assay kits are applied in the same experiment, the cost of the kits will be more than $500 \mathrm{RMB}$. As for the imported kits, they could cost more than $1000 \mathrm{RMB}$. The increase of cost is obvious.

The disadvantages for teachers. The kits make it possible that the experiment teacher will take a very short time to prepare an experiment. Even further, there is no experimental preparation and no pre-experiment. However, experimental preparation and pre-experiment are essential for the experimental teachers. Without preparation, they will rely heavily on the kits and omit pre-experiment, and even forget the skills they previously mastered of. So, the anaclisis on kit is not beneficial for teachers to enhance his experimental skill.

Besides, since the protocol of the assay kits are prepared so in detail that the teacher is not a guarantee for the success of experiment. The experiment is carried out strictly under the protocol, and the protocol usually gives little information beneficial for experimental theory education. For some commercial reasons, some assay kits could keep the information of the kit in secret, and the teacher can obtain almost no information on the principle of the kits. If the teacher does not know the principle of the kit, he can not explain the results his students gathered. In fact, an experiment like this is a thorough failure but possibly with perfect results. Even worse than this, if there are unknown defects in the kit, an expected result may be not obtained, and the teacher can do nothing but wait for the supplier's reply. The failure boils down to the kit, and the teacher can learn nothing technical from the event.

The disadvantages for students. The students are the operators of the assay kit. What they can do is to complete the procedure of the protocol strictly step by step with the hope to obtain the expected results. Thus, the principle of the procedure usually not addressed by the protocol is omitted by the students. Their goal is to harvest the expected results. So, the kit hampers the students from understanding the physicochemical properties of the reagent and the materials of the kit, and also hampers them from comprehending the principle of every step. The Kits make experimental operation foolproof, but help students to be a "fool" in the experiment, which is against the goal of experimental education.

\section{Overcoming the disadvantages of assay kits}

The assay kits are only an experimental tool, which is likely to resulting disadvantages listed above, and is not absolutely good or bad. There is no absolutely relationship between kit and experimental education in its possible disadvantages. If a teacher who recognized the disadvantages can actively take action, the disadvantages can be overcome. The disadvantages mostly caused by the unclear 
information of the standard operation protocol. If the unclear information becomes clear, the disadvantages will be overcome.

According to the authors' suggestion, we'd better avoid using kits in experimental education, especially, when experimental education is short of fund support. If application of assay kits is unavoidable, we should try our best to select kits for experimental education rather than those for scientific research or for clinic, though there could be few assay kits suitable for experimental education. When we must use assay kits for scientific research in experimental education, we should make a comprehensive preparation.

First, in order to insure the quality of the kit, the kit which will be used in experimental education should be tested by the teacher before students using it, and if not the experiment will run a risk. In one case, the authors were not able to obtain the expected results in a pre-experiment used assay kits. We found its reason was associated with the kit's quality, because the results were OK when a kit of new batch was applied.

Second, the experimental teachers must fully understand the kit, including its principle, physicochemical properties of the reagents and materials, and other information the protocol not addressed clearly. Thus, if there are something wrong with the experiment, the teachers are able to analyze the reason even solve the problem, rather than only wait for the supplier's reply. Even so, the students can still learn a lot from the experiment.

Third, the teachers should clearly explain the information of the kit to the students before or after the experiment. Especially, the relationship between the principle of the procedure and the possible results should be analyzed thoroughly as possible as they can. Of course, experimental education is one important part of science education, and the kit and the experiment should be partners of the theory education of the same curriculum.

Finally, the teachers should analyze the results from the kit. They should also compare the results with those from traditional methods obtained before. If there are odd results or the results deflecting from normal arrange, he should analyze them too.

\section{Conclusion}

The authors believe, if the teachers understand the benefits and the disadvantages of assay kits, and if they try their best to overcome the disadvantages, experimental education with assay kits will obtain expected results which are better than those obtained from classical methods. And the students can learn more.

\section{Acknowledgements}

The work was financial supported by Yunnan Provincial Science and Technology Department (1010CI043).

\section{References}

[1] B.G. Yan and J. Han: Journal of Shaanxi Institute of Education Vol. 24 (2008), p. 13

[2] D.K. Zhu: Experimental Technology and Management Vol. 25 (2008), p. 111

[3] B. Zhang and H.M. Guan: Research and Exploration in Laboratory Vol. 29 (2010), p. 135

[4] H.J. Jing, C.F. Zhang and C.X. Li: Journal of Biology Vol. 27 (2010), p. 100

[5] H. Xie, X.M. Shen: The Guide of Science \& Education Vol. 5 (2014), p. 196

[6] M.Y. Wu, S.Z. Xiong, X.D. Zhu, Y. Cao and C.K. Zhang, CN Patent 200510111813.9. (2006)

[7] Editor: Educational Equipment and Experiment Vol. 29 (2012), p. 34 
[8] X.J. Wang, L.J. Diao, X.H. Wang and G.P. Lv: Forensic Science and Technology Vol. 32 (2007), p. 52

[9] H.T. Xie, W.G. Chen and Y.H. Liu: China Medical Engineering Vol. 14 (2006), p. 199

[10] D. Wang, H.X. Song and L. He: Research and Exploration in Laboratory Vol. 28 (2009), p. 119 